\title{
Capítulo 1 Políticas de logística y cadena de suministro en México
}

\section{Chapter 1 Logistics and supply chain policies in Mexico}

PÉREZ-RAMÍREZ, Rigoberto $\dagger$

Universidad Autónoma del Estado de México. Centro Universitario UAEM Valle de México. Blvd. Universitario s/n Predio San Javier, Atizapán de Zaragoza, México, C.P. 54500

ID $1^{\text {er }}$ Autor: Rigoberto, Pérez-Ramírez / ORC ID: 0000-0003-2982-2676, Researcher ID Thomson: B-2673-2016, arXiv Author ID: rperezr, CVU CONACYT ID: 88934

DOI: $10.35429 /$ H.2020.1.1.14 


\title{
Resumen
}

El presente trabajo tiene como propósito analizar las políticas de logística y cadena de suministro en México a través de método descriptivo que nos permita hacer un recorrido histórico breve sobre la voz y uso frecuente del término logística por las organizaciones que cuentan con un gran número de puntos de suministro y de clientes dispersos geográficamente. Un ejemplo de ello, son las empresas transnacionales que en el contexto de la globalización llevan a cabo el aprovisionamiento de materiales, fabricación y distribución en distintos países. Las políticas de logística y cadena de suministro en México y sus empresas tienen que evolucionar en el mundo del comercio exterior pensando holísticamente y actuando de manera local que les permita competir y ganar mercados en una economía globalizada, contribuyendo de esta manera a un mejor desarrollo interno.

\section{Cadena de suministro, Logística, Globalización}

\begin{abstract}
The purpose of this work is to analyze the logistics and supply chain policies in Mexico through a descriptive method that allows us to make a brief historical tour of the voice and frequent use of the logistic term by organizations that have a large number of supply points and geographically dispersed customers. An example of this, is the transnational companies that in the context of globalization carry out the supply of materials, manufacturing and distribution in different countries. Logistics and supply chain policies in Mexico and its companies have to evolve in the world of foreign trade thinking holistically and acting in a local way that allows them to compete and win markets in a globalized economy, thus contributing to a better internal development.
\end{abstract}

\section{Supply chain, Logistics, Globalization}

\section{Introducción}

El presente trabajo tiene como propósito analizar las políticas de logística y cadena de suministro en México a través de método descriptivo que nos permita hacer un recorrido histórico breve sobre la voz y uso frecuente del término logística por las organizaciones que cuentan con un gran número de puntos de suministro y de clientes dispersos geográficamente. Un ejemplo de ello, son las empresas transnacionales que en el contexto de la globalización llevan a cabo el aprovisionamiento de materiales, fabricación y distribución en distintos países.

La globalización ha contribuido a la transformación de los sistemas logísticos de administración de la producción, favoreciendo a su vez la reestructuración de la organización territorial de la producción mundial a través del máximo aprovechamiento de las economías de escala; la obtención de ventajas competitivas de la especialización; la generación de oportunidades de racionalizar los costos; la creación de un consumidor homogéneo que, independientemente de su cultura, o de sus localización, comparte funciones de preferencias parecidas

La globalización exige ser capaz de coordinar actividades complejas, de formas que las compras, la producción y la financiación tengan lugar en los países con costos más bajos. Una perspectiva global de este tipo ha evidenciado la necesidad de gestionar la logística a nivel mundial. Específicamente, esta nueva logística debe ser capaz de controlar el proceso complejo de distribución de inversiones dentro y entre un gran número de naciones con leyes, culturas, niveles de desarrollo y aspiraciones diferentes.

En este contexto, el trabajo se divide en tres epígrafes: prolegómenos de la logística; del término a la polisemia conceptual de la logística y las políticas de logística y cadena de suministro en México, a fin de analizar y comprender la evolución de las políticas en una economía cada vez más interrelacionada e interdependiente. 


\section{Prolegómenos de la logística}

La voz logística proviene del latín logisticus "computación-cálculo", es decir, una serie de cálculos programados que se realizan para obtener un resultado. El término logística surge como tal a principios del siglo XX en referencia a una rama de la ciencia militar (Sun Tzu, 2005) ${ }^{1}$ que está relacionado con mantener y transportar material, suministros, personal e instalaciones que se requieren para una misión, en tanto que en materia comercial y, en un contexto previo al desarrollo industrial donde las actividades económicas predominantes eran la agricultura y la ganadería, el término logística permitía poner en contacto las nociones de oferta y demanda.

Durante la Segunda Guerra Mundial la logística comenzó a tener un aspecto empresarial como resultado del despliegue de las tropas estadounidenses que despertaron el interés del sector armamentista hacia nuevos mercados externos. De tal suerte, que a partir de la década de los sesenta, con base en los postulados del marketing, la logística adquirió su orientación actual: hacia el cliente. Pero no es sino hasta los ochenta cuando se utilizó ampliamente y donde las empresas comenzaron a tomar en cuenta las necesidades, requerimientos y requisitos de los clientes, y donde la logística logró un rol estratégico como generadora de ventajas competitivas. En este sentido, el empresario comienza a adoptar la logística integral (noción de costo total) y sus extensiones hacia el canal de aprovisionamiento, junto con los nuevos sistemas de gestión empresarial (producción flexible, Just in time, control de calidad, entre otros) y la reducción del costo de la tecnología de información.

Las empresas comprendieron que "la optimización de costes logísticos sería mayor si gestionaban de forma íntegra y unificada todas las actividades logísticas [...] como elementos interrelacionados que precisan de una gestión conjunta y desde una perspectiva global, desde el aprovisionamiento de materias primas hasta el cliente final" (Servera-Francés, 2010).

Para la década de los noventa, la logística adquiere mayor relevancia dentro de la gestión empresarial, poniendo énfasis en las alianzas estratégicas con los proveedores. A la sazón de ello, la logística gira en torno a la creación de valor, es decir, valor para los clientes, los proveedores y los accionistas de la empresa, que se materializa en ofrecer un servicio logístico acorde con los requisitos del cliente. Aquí se identifica un creciente interés en el estudio de la integración logística a lo largo de todo el canal de suministro con el fin de ofrecer un mayor valor al cliente (Servera-Francés, 2010).

En 1998, el Council of Logistics establece una nueva definición de logística, en la cual aparece el término de "flujo inverso", que hace referencia a lo que posteriormente se conocerá como Logística Reversa o Inversa. Se trata de la gestión de retornos que fluyen desde el consumidor al fabricante.

Lo anterior, condujo al Council of Logistics Management, en 2005, cambiar su nombre por el de Council of Supply Chain Management Professionals. A partir de entonces, "la dirección de logística de los negocios se conoce ahora popularmente como dirección de cadena de suministros. Se usan otros términos, como redes de valor, corrientes de valor y logística ágil para describir un alcance y un propósito parecidos" (Ballou, 2005). Se trata de una visión integradora de la logística que avanza junto a la actual economía mundial integradora que caracteriza a la globalización. De ahí que la logística se defina como aquella parte de la Supply Chain que planifica, implementa y controla desde el punto de origen al punto de consumo con el objetivo de satisfacer los requerimientos del cliente (Council Supply Chain Management Professionals, 2014). Esta definición incluye los conceptos de "flujo directo e inverso", que hacen referencia a los conceptos de logística directa y logística inversa. La logística directa es relacionada con la logística tradicional, como se observa en la Figura 1.1.

\footnotetext{
${ }^{1}$ La referencia más antigua de la logística militar aparece entre los años 2900 y el 2800 A.C, como la organización del ejército, las graduaciones y rangos entre oficiales, la regulación de las rutas de suministros y la provisión de material militar del ejército.
} 
Figura 1.1 Flujos de la logística directa

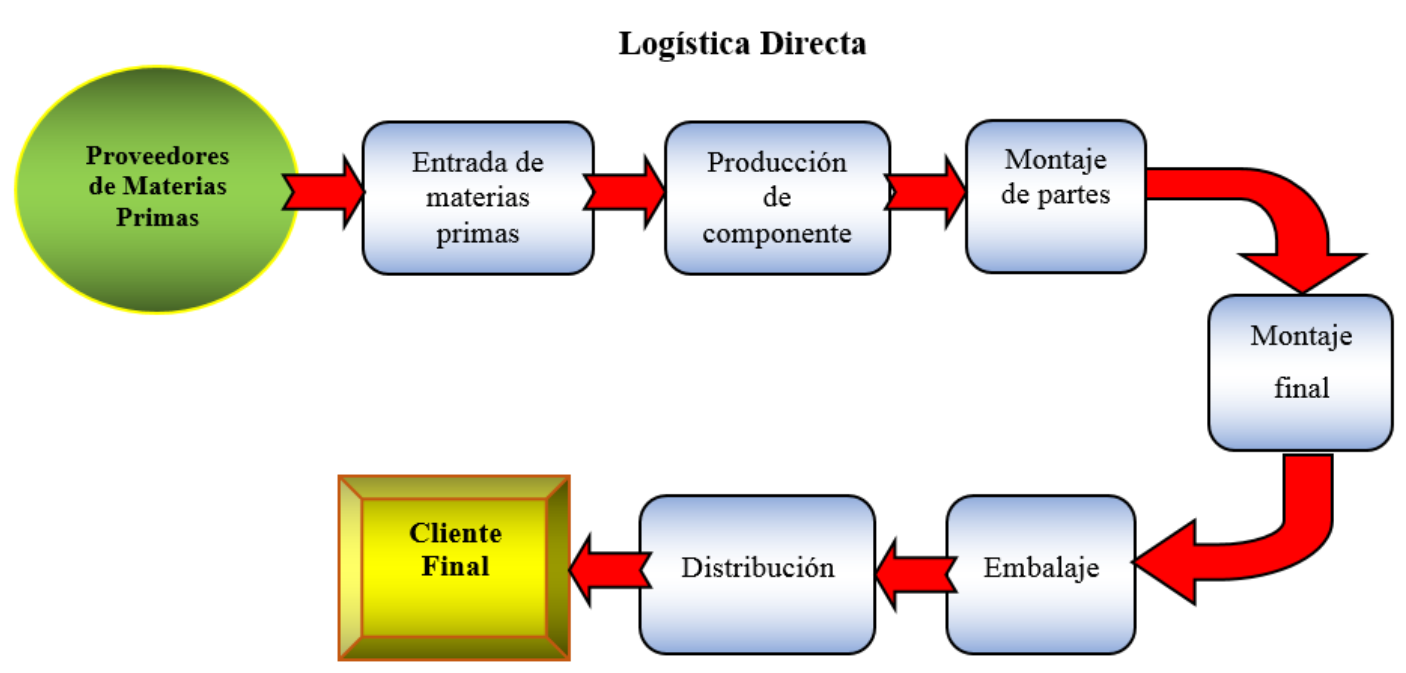

Fuente: Elaboración Propia

Como se puede observar en la figura anterior, la logística directa comprende una serie de procesos que parte de la línea de proveedores de materias primas hasta el cliente final pasando por la entrada de materias primas, producción de componentes, montaje de partes, montaje final, embalaje y distribución.

Ahora bien, la empresa moderna cada vez más recupera productos o materiales de los clientes, ya sea para recuperar valor o bien como servicios de postventa. A este proceso se le denominó logística inversa $^{2}$ (Luttwak, 1971), también conocida como "la cadena de suministro inversa", donde los fabricantes diseñan procesos para reusar sus productos (Guide, 2002). En este sentido, la logística inversa se define como el proceso de planificación, ejecución y control de la eficiencia y eficacia del flujo de las materias primas, inventario en proceso, productos terminados e información relacionada desde el punto final (consumo) hasta el punto de origen, con el fin de recuperar valor o la correcta eliminación (Rogers \& Tibben-Lembke, 1999).

Dentro de las operaciones que pueden enmarcarse en la logística inversa se encuentran: los procesos de retorno de excesos de inventarios, devoluciones de clientes, productos obsoletos, sobrantes de demandas estacionales, inventarios, entre otros. Así como actividades de retirada, clasificación, reacondicionamiento y reenvío al punto de venta a otros mercados secundarios. Mediante la logística inversa cuando un producto se ha devuelto a la empresa, ya se trate de una devolución dentro del período de garantía o de un producto al final de su vida útil, la empresa dispone de diversas formas de gestionarlo con vistas a recuperar parte de su valor, que puede darse en cada uno de los pasos, como se puede observar en la siguiente figura.

La figura de la logística inversa muestra el proceso de retorno en cada una de las etapas para recuperar parte del valor en materias primas., producción ce componentes, manejo de partes, embalaje y distribución, antes de llegar al cliente final.

\footnotetext{
${ }^{2}$ La logística inversa también recibe el nombre de distribución inversa, retrologística, logística de la recuperación y el reciclaje.
} 
Figura 1.2 Flujos de la logística Directa e Inversa

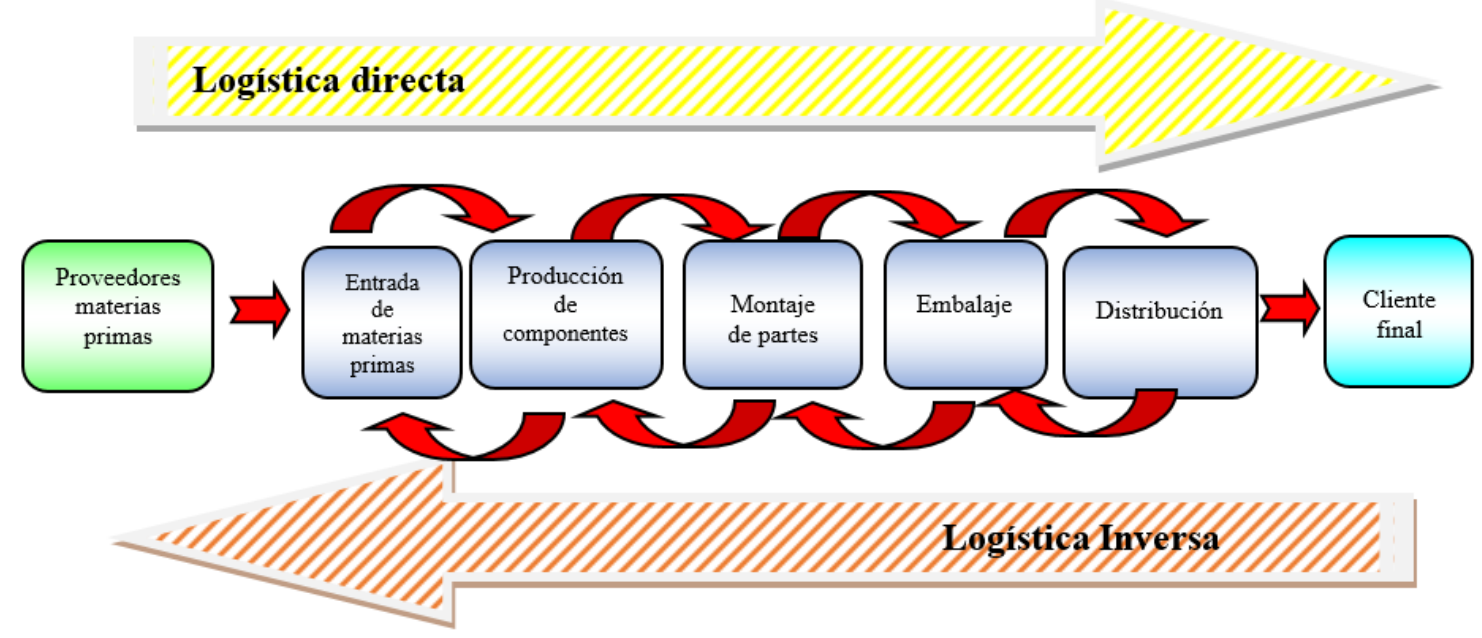

Fuente: Elaboración Propia

En la actualidad es más frecuente el uso del término logística por las organizaciones que cuentan un gran número de puntos de suministro y de clientes dispersos geográficamente. Un ejemplo de ello, son las empresas transnacionales que en el contexto de la globalización llevan a cabo el aprovisionamiento de materiales, fabricación y distribución en distintos países. La distribución logística se unió a la gestión de materiales la cual adoptó rápidamente una postura proactiva en el diseño de la estrategia de fabricación. Así, las empresas multinacionales en la globalización se distinguen por su capacidad de integrar y controlar operaciones internacionales, con fabricación especializada y estrategias de mercadeo global. En este sentido, la globalización exige ser capaz de coordinar actividades complejas, de formas que las compras, la producción y la financiación tengan lugar en los países con costos más bajos. Una perspectiva global de este tipo ha evidenciado la necesidad de gestionar la logística a nivel mundial. Específicamente, esta nueva logística debe ser capaz de controlar el proceso complejo de distribución de inversiones dentro y entre un gran número de naciones con leyes, culturas, niveles de desarrollo y aspiraciones diferentes. Por ejemplo. McDonald’s (Mora, 2008: 27). De ahí, que la logística recurre a varias actividades y Know How que participan en la gestión y control de los flujos físicos, de informaciones y medios. Una síntesis de la evolución histórica de la logística se muestra el siguiente cuadro sinóptico.

Tabla 1.1 Evolución histórica de la logística

\begin{tabular}{|c|c|c|}
\hline Periodo & Denominación & Principales características \\
\hline 1. $1916-1940$ & Del campo al mercado & $\begin{array}{ll}\checkmark & \text { Del campo al mercado } \\
\checkmark & \text { Almacenamiento } \\
\checkmark & \text { Transportación }\end{array}$ \\
\hline 2. $1940-1960$ & Funciones segmentadas & $\begin{array}{ll}\checkmark & \text { Áreas funcionales independientes } \\
\checkmark & \text { Transportación in-bound y out-bound } \\
\checkmark & \text { Inventario al por mayor } \\
\checkmark & \text { Distribución física }\end{array}$ \\
\hline 3. $1960-1970$ & Funciones integradas & $\begin{array}{ll}\checkmark & \text { Costo total } \\
\checkmark & \text { Enfoque de Sistema } \\
\checkmark & \text { Integración de la logística }\end{array}$ \\
\hline 4. 1970-mitad de 1980 & Enfoque al cliente & $\begin{array}{ll}\checkmark & \text { Mantenimiento del inventario } \\
\checkmark & \text { Productividad } \\
\checkmark & \text { Redes vinculadas }\end{array}$ \\
\hline $\begin{array}{l}\text { 5. Mitad de } 1980 \text { hasta } \\
1996\end{array}$ & $\begin{array}{lll}\text { Logística } & \text { como } & \text { factor } \\
\text { diferenciador } & & \end{array}$ & $\begin{array}{ll}\checkmark & \text { Suministro integrado } \\
\checkmark & \text { Canal logístico } \\
\checkmark & \text { Globalización } \\
\checkmark & \text { Entorno logístico } \\
\checkmark & \text { Tecnologías de la información y las comunicaciones }\end{array}$ \\
\hline $\begin{array}{l}\text { 6. } 1996 \text { hasta } \\
\text { el presente }\end{array}$ & $\begin{array}{l}\text { Expansión de las fronteras y la } \\
\text { actividad }\end{array}$ & $\begin{array}{ll}\checkmark & \text { Logística de respuesta al servicio } \\
\checkmark & \text { Comportamiento intercompañías } \\
\checkmark & \text { Dirección integrada de la cadena de suministro } \\
\checkmark & \text { Desarrollo de la teoría } \\
\checkmark & \text { Logística inversa }\end{array}$ \\
\hline
\end{tabular}




\section{Del término a la polisemia conceptual de logística}

La logística es un concepto polisémico dada la infinidad de definiciones que pueden ser analizadas desde diferentes connotaciones. Por ejemplo, para el Diccionario de la Real Academia Española (RAE, 2018), la logística es "conjunto de medios y métodos necesarios para llevar a cabo la organización de una empresa, o de un servicio, especialmente de distribución". Por su parte, Ballou, define la logística como "todo movimiento y almacenamiento que facilite el flujo de productos desde el punto de compra de los materiales hasta el punto de consumo, así como los flujos de información que se ponen en marcha, con el fin de dar al consumidor el nivel de servicio adecuado a un costo razonable" (Ballou, 1999: 7).

Otros autores, como Adriaenséns y Flores, argumentan que la logística es "una función operativa importante que comprende todas las actividades necesarias para la obtención y administración de materias primas y componentes, así como el manejo de los productos terminados, su empaque y su distribución a los clientes" (2004: 282). Para Lamb, Hair y MacDaniel, la logística es un "proceso de administrar estratégicamente el flujo y almacenamiento eficiente de las materias primas, de las existencias y de los bienes terminados del punto de origen al de consumo" (2002: 383).

En tanto, Waters, sostiene que la logística puede ser considerada como un proceso integrativo que busca optimizar el flujo de productos e información a través de la organización y la operación hacia el consumidor (Waters, 2007: 21). Para Castellanos, la importancia de la logística reside en la necesidad de mejorar el servicio a un cliente, optimizando la fase de mercadeo y transporte al menor costo posible (Castellanos, 2009: 6). Entre las mejoras que se pueden encontrar están: la cadena de distribución debe mantener cada vez menos inventarios, niveles altos en la eficiencia en producción y desarrollo de sistemas de información. Todo ello, para lograr los siguientes beneficios: a) incremento en la competitividad y mejora de la rentabilidad para superar las condiciones del mercado global, b) optimización de la gerencia y la gestión logística comercial nacional e internacional, y c) ampliación de la visión gerencial para convertir la logística en un mecanismo para la planificación de las actividades internas y externas de la empresa (Zuluaga, Gómez \& Fernández; 2014)

En este sentido, la logística es el "proceso integral que busca anticipar los requerimientos de los clientes, adoptando y administrando estratégicamente los recursos necesarios para asegurar la distribución de bienes, información y servicios hasta el cliente final, de forma completa, oportuna y a un costo justo. De tal manera que el objetivo de agregar valor se cumpla" (Franco, 2008: 71). Siguiendo a Franco "todas aquellas actividades que involucran el movimiento de materias primas, materiales y otros insumos, así como, todas aquellas tareas que ofrecen un soporte adecuado para la transformación de dichos elementos en productos terminados tales como las compras, el almacenamiento, la administración de los inventarios y los suministros, forman parte del proceso logístico. De ahí, que se entienda la logística como una cadena formada por tres eslabones principales que son abastecimiento, la producción y la distribución" (Franco, 2008: 71-72).

Como se puede observar, la logística es una actividad interdisciplinaria que vincula las diferentes áreas de la empresa, desde la programación de compras hasta el servicio postventa; pasando por el aprovisionamiento de materias primas; la planificación y gestión de la producción; el almacenamiento, manipuleo y gestión de stock, empaques, embalajes, transporte, distribución física y los flujos de información (Mora, 2008). Todo ello, hace que la logística se conozca como dirección de cadena de suministros, en otras palabras, la logística queda comprendida dentro de la cadena de suministro formando parte de ésta.

La cadena de suministro o supply chain (Figura 1.3) se encuentra constituida por instalaciones geográficamente dispersas por todo el mundo, donde la materia prima, los productos en proceso y los productos terminados han de ser adquiridos, transformados, ensamblados, almacenados y vendidos. 
Figura 1.3 Cadena de suministro

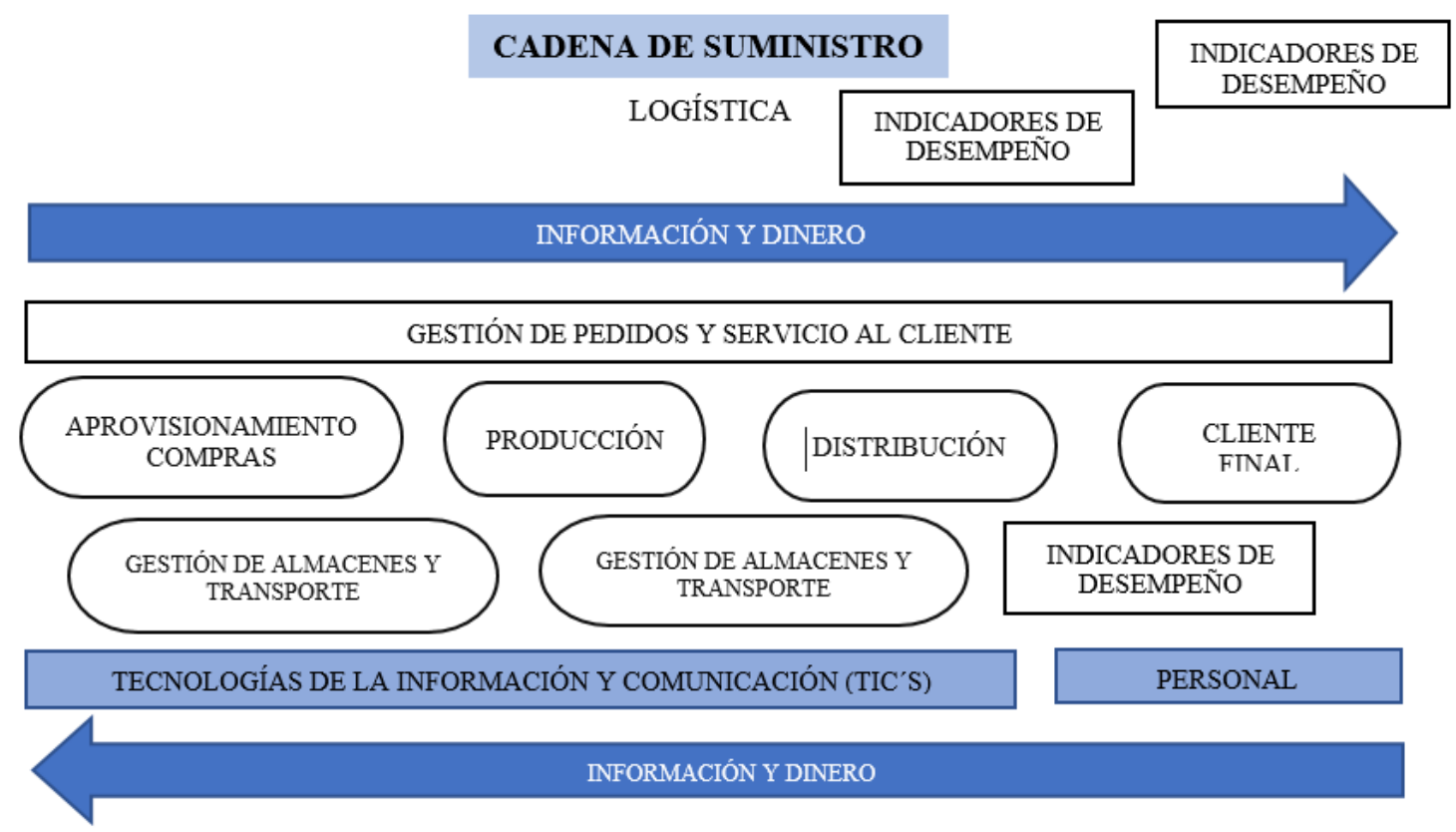

Fuente: Zuluaga, Gómez \& Fernández (2014: 95)

Como se ilustra en la figura, la cadena de suministro a través del empleo de las tecnologías de la información y comunicación genera la gestión de pedidos y servicio al cliente final teniendo presente indicadores de desempeño en la gestión de almacenes y transportes que tienen que ver con aprovisionamiento de compras, producción y distribución a través del retorno de información y dinero.

En la actualidad el mercado global impone todas las empresas esquemas de competencia entre cadena de suministros, en otras palabras, la competencia tradicional basada en productos ya no es suficiente para ganar los mercados y satisfacer a los clientes, quienes son cada vez más exigentes y quieren obtener mayores beneficios por parte de las empresas. En este sentido, para los clientes actuales, el valor que perciben de las empresas no sólo está en sus productos y servicios, sino en la forma en la que se ofrecen: en la agilidad, en la flexibilidad, en la capacidad de respuesta, en el nivel de servicio, en ña personalización del producto o servicio, en la atención preventa y postventa, y en una infinidad más de elementos de distinta índole (Romero \& Elizondo, 2015: 127). De tal suerte, la cadena de suministro muestra un sistema complejo (Ackoff, 2010; Wolfram, 2002), cada vez mayor en sus distintas dimensiones, presentando un enorme número de elementos vinculados por múltiples relaciones no lineales, y sobretodo, que presentan comportamientos emergentes (Boccara, 2004), que además, cambian en el tiempo (Romero \& Elizondo, 2015).

El enfoque sistémico constituye una forma de abordar, representar y aproximar los distintos niveles de la realidad en la que nos encontramos. Su evolución a través del tiempo, adaptándose a las nuevas realidades, hace posible su aplicación a los más diversos problemas, de manera que el estudio de la cadena de suministro no ha sido la excepción y ha permitido el desarrollo de un marco de referencia para la toma de decisiones.

En la actualidad, el concepto de cadena de suministro en el mercado global considera que las empresas se encuentran dentro de un ambiente altamente competitivo donde las operaciones se extienden hacia el exterior de las empresas y de las naciones de donde son originarias, en dos sentidos: buscando proveedores que agregan valor a la cadena de suministro y buscando los mercados que han de ser capturados económicamente y que representan las mejores oportunidades de generar utilidades. De ahí, que el objetivo global de la cadena de suministro es generar las mayores utilidades -minimizando costos y maximizando ingresos-. Por ello, la generación de empleos (directos e indirectos), la, productividad, la eficiencia, entre otros, no son los objetivos primordiales de las empresas globales ni de la cadena de suministro, son elementos que se requieren para alcanzar el objetivo global y, por ende, se deben encontrar subordinados a éste. 
La globalización ha contribuido a la transformación de los sistemas logísticos de administración de la producción, favoreciendo a su vez la reestructuración de la organización territorial de la producción mundial a través del máximo aprovechamiento de las economías de escala; la obtención de ventajas competitivas de la especialización; la generación de oportunidades de racionalizar los costos; la creación de un consumidor homogéneo que, independientemente de su cultura, o de sus localización, comparte funciones de preferencias parecidas (Jiménez \& Hernández, 2002).

En este contexto, México y sus empresas tiene que evolucionar en las políticas de logística y cadena de suministro en el comercio exterior, pensando global y actuando localmente para competir en una economía cada vez más interrelacionada e interdependiente. Dicha interdependencia se consolida debido a la mayor integración de los sistemas de producción fragmentados ${ }^{3}$ manejados a escala mundial por múltiples empresas transnacionales ${ }^{4}$.

\section{Políticas de logística y cadena de suministro en México}

En México, los servicios en el comercio son factores clave para el crecimiento y el aumento de la competitividad en este ámbito. La cercanía geográfica de las economías de México y Estados Unidos y sus ventajas comparativas permitieron complementarse consolidando su integración económicacomercial con la entrada en vigor el 01 de enero de 1994, el Tratado de Libre de América del Norte (TLCAN) $)^{5}$ entre Canadá, México y Estados Unidos.

Es en 1983, cuando México firmó una Carta de Intención con el Fondo Monetario Internacional (FMI), en la que se comprometía llevar a cabo una revisión de los sistemas de protección arancelaria e iniciar procesos de apertura comercial. A partir de entonces, la apertura comercial ha significado una demanda creciente del sector exportador e importador del país, así como de otros sectores productivos y de servicios relacionados.

Desde de la logística, esto no sólo ha demandado el simple traslado o arrastre de carga entre países -transporte transfronterizo, de larga distancia y/o local-, sino también implicado el establecimiento de estrategias eficiente gestión de las cadenas de suministro involucrando tanto el sector público como privado (productores, proveedores y comercializadores), lo que ha implicado el desarrollo de mecanismos, procesos, capacidades y recursos varios con el propósito de ir transformando la logística mexicana en una disciplina más competitiva, productiva, integrada y especializada (Murguía, 2014)

De tal manera, que durante el periodo del TLCAN comenzaron a fortalecerse algunos factores que son considerados base de la evolución del sector logístico en la región de América del Norte.

Tabla 1.2 Factores el sector logístico en América del Norte

\begin{tabular}{|l|l|}
\hline Factor & Propósito \\
\hline Infraestructura & $\begin{array}{l}\text { Desarrollo de estructuras como bodegas, naves industriales, almacenes, puertos marítimos, vías y } \\
\text { carreteras, terminales intermodales y aeropuerto para potenciar las actividades relacionadas a } \\
\text { cadenas de suministro, generando plataformas logísticas que faciliten la implementación de } \\
\text { actividades comerciales y entregando mejores condiciones a las empresas que se dedican a la } \\
\text { logística y transporte de productos a lo largo del país. La infraestructura ayuda a la productividad } \\
\text { a través del apoyo de nuevas tecnologías y es impulsada desde el sector privado con la apertura al } \\
\text { desarrollo por parte del sector público, lo que ha permitido a lo largo de los años atraer una mayor } \\
\text { inversión extranjera. }\end{array}$ \\
\hline
\end{tabular}

\footnotetext{
${ }^{3}$ Como resultado de la fragmentación de producción se origina automáticamente un proceso intenso de terciarización "outsourcing" de la producción, intensificando las redes empresariales integradas por medio de cadenas de logísticas de suministro.

${ }^{4}$ las empresas transnacionales son sociedades que poseen y controlan medios de producción o servicios fuera del país en el que están establecidas.

${ }^{5}$ El TLCAN ha sido renegociado por los gobiernos de México, Estados Unidos y Canadá y denominado en 2018 como TMEC tras su aprobación por los poderes ejecutivos, respectivamente. Sin embargo, hoy en día sólo el congreso mexicano ha secundado dicha aprobación, falta el congreso de Estados Unidos y Canadá, para que pueda entrar en vigor.
} 


\begin{tabular}{|c|c|}
\hline Tecnología & $\begin{array}{l}\text { La tecnología es piedra angular de la empresa, el desarrollo de los sistemas de información más } \\
\text { especializados y confiables (WMS }{ }^{6} \text {, TMS }{ }^{7} \text {, YMS }{ }^{8} \text {, GPS }{ }^{9} \text {, etc.), las redes que soportan la } \\
\text { infraestructura, la transmisión segura de datos, la conectividad con los sistemas de los clientes y } \\
\text { con la base de proveedores son algunas de las cosas que han empujado a todos los participantes a } \\
\text { mejorar la calidad de sus servicios, ofreciendo soluciones que garanticen mayor eficacia y } \\
\text { seguridad a los clientes }\end{array}$ \\
\hline Certificaciones & $\begin{array}{l}\text { Aumenta la satisfacción de sus clientes, a mejorar costos, a tener un valor mayor en sus productos } \\
\text { (Lean Six Sigma }{ }^{10} \text {, ISO }{ }^{11} \text {, C-TPAT }{ }^{12} \text {, FAST }{ }^{13} \text {, etc.). Además de proveer de credibilidad y } \\
\text { confianza a los socios de la empresa, también permiten el desarrollo de otro sector de la industria, } \\
\text { ya que hay muchas empresas que están atrás soportando el éxito de ellos al permitirles tener y } \\
\text { mantener sus certificaciones. }\end{array}$ \\
\hline $\begin{array}{l}\text { Creación de } \\
\text { asociaciones }\end{array}$ & $\begin{array}{l}\text { Muchas organizaciones relacionadas con la logística se han ido conformado en los últimos } 20 \text { años, } \\
\text { y son quienes representan los intereses de los jugadores del gremio logístico y quienes ayudan a } \\
\text { regular negociaciones entre el sector público y el privado, participando en acciones que benefician } \\
\text { el funcionamiento de la logística en el país, como por ejemplo, la Asociación Mexicana de } \\
\text { Operadores Logísticos. }\end{array}$ \\
\hline Educación logística & $\begin{array}{l}\text { En } 1994 \text { en México no había formación universitaria que abordara temas de logística específicos, } \\
\text { la educación existía parcialmente y en un nivel más técnico. Sin embargo, el aumento en la } \\
\text { demanda de servicios de este tipo ha generado una evolución en la educación relacionada con este } \\
\text { sector. Hoy en día, se pueden encontrar planes de estudio a nivel licenciatura o maestría, así como } \\
\text { diplomados especializados en temas de logística y transporte, lo que ha permitido una } \\
\text { profesionalización de los servicios en gran parte del sector logístico. }\end{array}$ \\
\hline $\begin{array}{l}\text { Cultura del } \\
\text { outsourcing y } \\
\text { consultoría }\end{array}$ & $\begin{array}{l}\text { Otro factor que también ha crecido en los últimos } 20 \text { años permite la tercerización de los servicios } \\
\text { (3PL's y 4PL's o LLP'14), herramienta que está ascendiendo y entiende las necesidades de las } \\
\text { empresas actuales, jugando un papel muy importante en el mundo de la logística a nivel global. }\end{array}$ \\
\hline Servicios logísticos & $\begin{array}{l}\text { La oferta de servicios y soluciones que proveedores logísticos ofrecen en el mercado mexicano ha } \\
\text { contribuido a trasladar beneficios a sus clientes en términos de costos y mejora en los niveles de } \\
\text { atención al cliente a través de la excelencia y experiencia operativa. }\end{array}$ \\
\hline
\end{tabular}

Fuente: Elaboración propia con base a Murguía (2014)

Cabe mencionar, que la tercerización de los procesos logísticos comenzó a desarrollarse entre 1980 y 1995, por medio de Third- Party-Logistics (3PL's), los cuales son operadores logísticos que proveen servicios básicos, algunos ofrecen servicios de manejo de información y cuentan con alta cobertura geográfica y escasa especialidad funcional, con la ventaja de poder operar en varias industrias (Figura 1.4).

\footnotetext{
${ }^{6}$ Warehouse Management System (WMS), es un Sistema que contribuye a la administración del suministro de la materia prima en los almacenes, además de controlar las órdenes de pedido de los clientes. Permiten contar con información en tiempo real sobre el nivel de utilización y rotación de la materia del almacén con lo que se reducen los costos de optimización de procesos, ayuda en un mejor nivel de servicio y reduce de manera significativa los tiempos de entrega.

${ }^{7}$ Transportation Management System (TMS), analizan las mejores alternativas de transporte y rutas para optimizar los costos y tiempos de traslado.

${ }_{8}$ Yard Management System (YMS) es un sistema de administración de patios que permite controlar los muelles de recepción y despacho, el rastreo y seguimiento del movimiento de los camiones a través de tecnología de localización en tiempo real.

${ }^{9}$ Global Positioning System (GPS) es un sistema de navegación que permite determinar la posición de cualquier objeto (persona, vehículo) en tiempo real con una precisión de centímetros.

${ }^{10}$ Es un enfoque para la mejora empresarial que se centra en la reducción de las variaciones en todos los procesos de trabajo. La reducción sistemática de variación genera procesos de alta calidad, que a su vez, suponen una mejora de la satisfacción de los clientes y mayores beneficios.

${ }^{11}$ Es la Organización Internacional de Normalización, cuya principal actividad es la elaboración de normas técnicas empresariales.

${ }^{12}$ Customs-Trade Partnership Agains Terrorism (C-TPAT) es una iniciativa entre el gobierno de los Estados Unidos y la iniciativa privada con la finalidad de garantizar la seguridad en las distintas áreas de la cadena de suministro y la seguridad en las fronteras. La C-TPAT surgió a raíz de los atentados del 11 de septiembre de 2001 en Nueva York como un esfuerzo del gobierno estadounidense por incrementar la seguridad fronteriza.

${ }^{13}$ Es conocer los procesos de la empresa de forma expedita que permita tener un panorama general en corto tiempo, un promedio de cuatro semanas, dependiendo de la complejidad de los procesos internos y externos de la empresa y el alcance que se establezca.

${ }^{14}$ En la etapa inicial Lead Logistic Provider (LLP) debía ser capaz de diseñar una solución logística óptima lo cual implicaba entender la cadena de suministro, los niveles de servicios requeridos y la estructura de costos. También debía definir los responsables de ejecutar los servicios los cuales los podía realizar directamente o bien a través de otros proveedores logísticos como Third-Party-Logistics (3PL's), Fourth-Party-Logistics (4PL's).
} 
A partir de 1995, surgieron los servicios de tercerización ofrecidos por Fourth-Party-Logistics (4PL's), los cuales son considerados un socio de la cadena de suministro, pues poseen alto conocimiento del negocio del cliente, cuentan con un alto compromiso con base a objetivos comunes, tiene capacidad de innovación, alta inversión tecnológica que abarca a toda la cedan de suministro y aplican las mejores prácticas globales (Figura 1.5). De tal manera, que ambos tipos de servicios logísticos atendían a la demanda de los clientes y a los retos que enfrentaban en su momento (USAID/México, 2014: 31).

Figura 1.4 Relación de los 3PL en la cadena de suministro

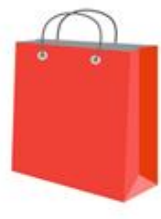

Proveedores

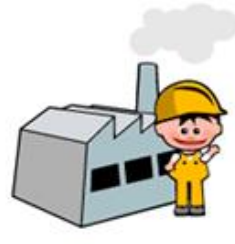

Manufactura

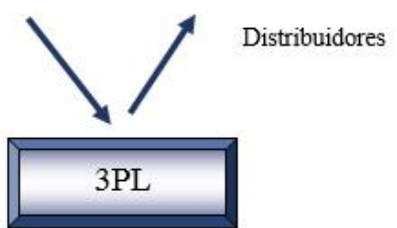

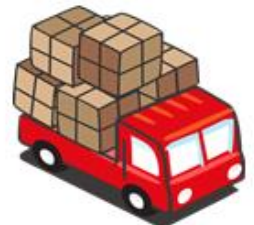

istribuidores

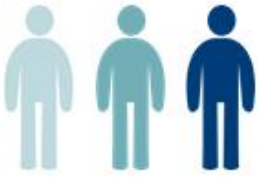

Consumidores

Fuente: USAID/México (2014: 32)

Nota: Los 3PL reciben el producto de las empresas que los contratan, desarrollan sus actividades y entregan a los clientes de estas empresas.

La figura 1.4 describe la terciarización de los procesos logísticos a través de los proveedores, la manufactura y los distribuidores para la cadena final representada por los consumidores. Por su parte en la figura 1.5, los servicios de terciarización ofrecidos por la 4PL's permite mejorar dada su alta inversión tecnológica y capacidad de innovación, la cadena de suministro representado por los proveedores, la manufactura, el transporte multimodal y los distribuidores para llegar a los consumidores.

Figura 1.5 Relación de los 4PL en la cadena de suministro

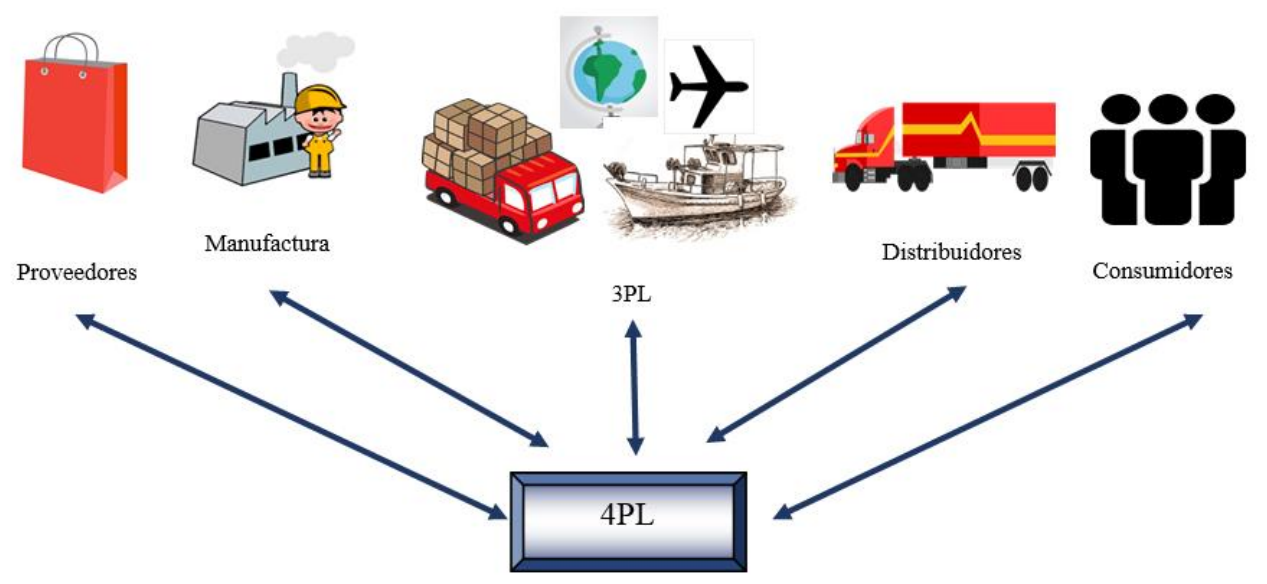

Fuente: USAID/México (2014: 32)

Nota: Los 4PL necesitan mantener relaciones fuertes con la mayoría de los actores de la cadena, funcionando como una torre de control para todos los participantes.

En general, para los especialistas los servicios logísticos han incorporado un gran valor agregado gracias a las tecnologías de información y comunicación por medio de actividades de planificación y sistemas de última generación, pues los servicios de los proveedores logísticos se encuentran presentes a lo largo de toda la cadena de suministro (figura 1.6). 
Figura 1.6 Presencia de los servicios otorgados por los proveedores de logística a lo largo de la cadena de suministro
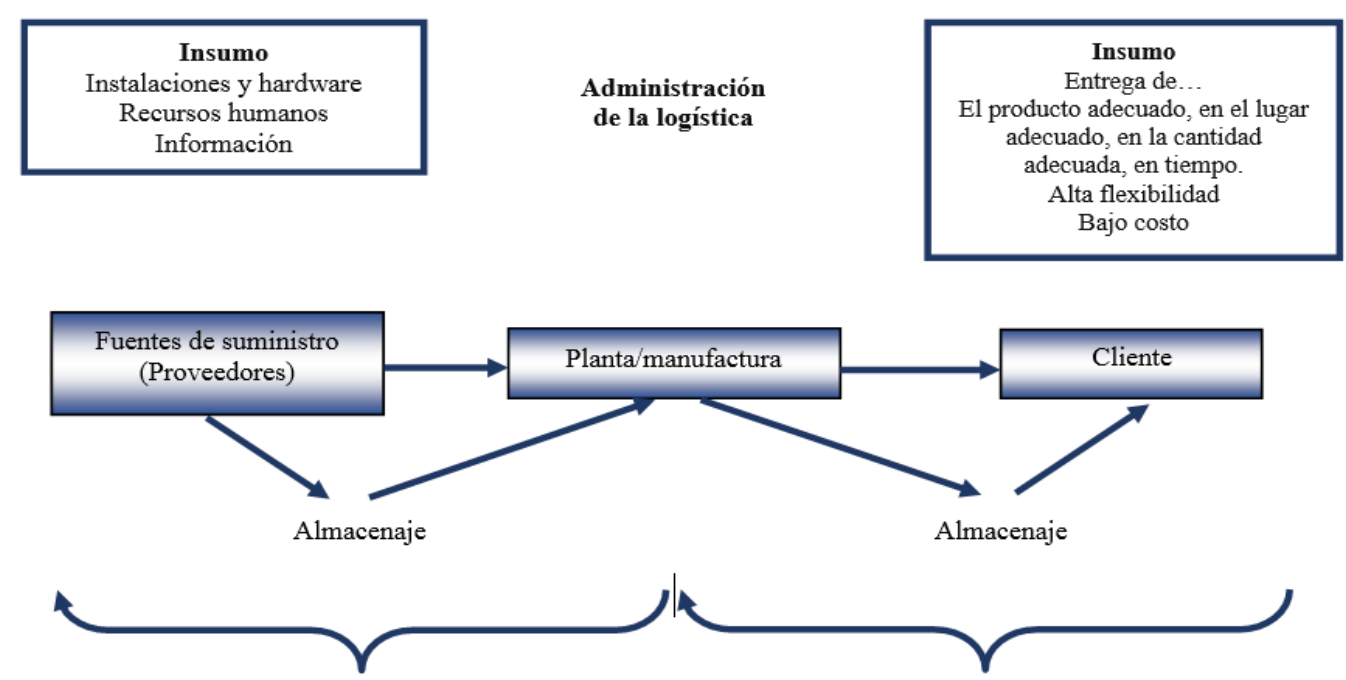

Fuente: USAID/México (2014: 35)

La figura 1.6 muestra la logística de abastecimiento y logística de entrega. La primera integrada por la fuente de suministro (proveedores) y la planta/manufactura que comprende los insumos (instalaciones, hardware, recursos, información y recursos financieros. La segunda está compuesta por la planta/manufactura y el cliente cuyos insumos son la entrega del producto adecuado, en el lugar adecuado, en la cantidad adecuada, en tiempo y a bajo costo. Todo ello, bajo un proceso de retroalimentación de ambas logísticas enmarcado en la visión holística de la administración logística. En la actualidad se pueden incluir en los factores del sector logístico: a) análisis y diseño de flujos de insumos y productos en las cadenas de suministro, b) diseño y operación de redes de distribución y administración de servicios de transporte -arrendamiento, terceros, multimodal, entre otros-, c)consolidación de embarques y programación de embarques y programación de servicios, d) integración de servicios transfronterizos -transporte cruce, aduana, documentación, sistemas de seguridad, patio, entre otros-, e) administración de inventarios (incluyendo programas de VMI), f) almacenamiento (diseño, ubicación y operación de los almacenes, y cross docks), g) procesamiento de pedidos, h) actividades des valor agregado (kitting, etiquetado, maquila, postponement, selección y reemplazo de componentes, documentación y equipos, compras, embalaje, entre otros), i) manejo de materiales, j) logística inversa, k) programación de producción (secuenciación y alimentación a líneas de producción), y l) tecnología de información, incluyendo recolección, procesamiento, análisis de datos e integración de sistemas, por mencionar algunos (Murguía, 2014).

Dentro del crecimiento de la logística en México ésta se ha orientado a la apertura del mercado orientada a la globalización lo que permite mayor competitividad y mejora en los niveles de servicio. El fenómeno del nearshoring en el que las empresas prefieren tener sus fábricas en otros mercados cercanos a sus clientes están dejando de producir en lugares como China por su mano de obra barata, trayendo sus plantas a países situados en zonas más cercanas a sus consumidores principales como México, el cual cuenta con una alta calidad en servicios de manufacturación, que genera confianza e inversión (Murguía, 2014). Sin embargo, especialistas en el tema consideran que existen muchos retos que la logística doméstica e internacional tiene que enfrentar tales como: i) visibilidad de principio a fin en las cadenas de suministro globales, ii) seguridad de la mercancía y de los activos, iii) colaboración y sincronización entre cadenas de suministro globales, iv) cambio climático y energías renovables, v) crecimiento y ampliación de las tecnologías de información, vi) mejora continua en las condiciones y características de la infraestructura física (puertos, aeropuertos, carreteras federales, caminos y vialidades locales, entre otros), vii) adecuación y cumplimiento de las leyes y reglamentos para una logística competitiva, integral y eficiente, entre otras. En este contexto, un estudio comparativo de las prácticas y tendencias en México, sobre la eficiencia de la cadena de suministro, realizado en 2017 y analizada por Deloitte considera que: a) las empresas que logren mejoras sustanciales en su planeación de demanda destacarán dentro de su industria, dado que el 69\% de las empresas identifican que la planeación de la demanda es uno de los principales restos en la cadena de suministro. 
Sin embargo, solamente las empresas medianas ponen la planeación y pronóstico como una iniciativa de mejora de alta prioridad en los próximos tres años; b) la capacitación y el desarrollo humano deben tomar un rol más más relevante en las empresas, pues el $61 \%$ de ellas identifica que la mano de obra calificada es uno de los principales restos de la cadena de suministro. Pero solamente la industria logística coloca la capacitación y desarrollo de su personal como prioridad de su plan en os siguientes tres años; c) las inversiones y mejoras en procesos y tecnologías seguirán siendo las iniciativas predominantes, ya que el $25 \%$ de los ejecutivos ha implementado tecnología para mejorar su cadena de suministro. Las empresas grandes dan prioridad a sistemas de tecnología mientras, que las medianas y pequeñas lo incorporan progresivamente como parte de su plan en los próximos tres años. El $60 \%$ de los encuestados considera que aún no es totalmente satisfactorio lo que ha implementado; d) todas las partes de la cadena de suministro son tercerizables, las empresas que mejor definan su estrategia podrán tener beneficios diferenciales. En promedio el $47 \%$ de las empresas ha tercerizado algún área de su logística como parte de su estrategia enfocándose a su negocio principal, sin embargo, el 35\% de los ejecutivos muestran incertidumbre en la tercerización de las áreas (Deloitte, 2017). De acuerdo con la encuesta de Deloitte (2017), las empresas grandes, medianas y pequeñas se han dado a la tarea de realizar un plan de implementación de iniciativas en el que sus prioridades dependen de su grado de participación en el mercado y tamaño de la empresa, respectivamente, como se muestra en la siguiente figura.

Figura 1.7 Plan de implementación de iniciativas en los próximos 3 años

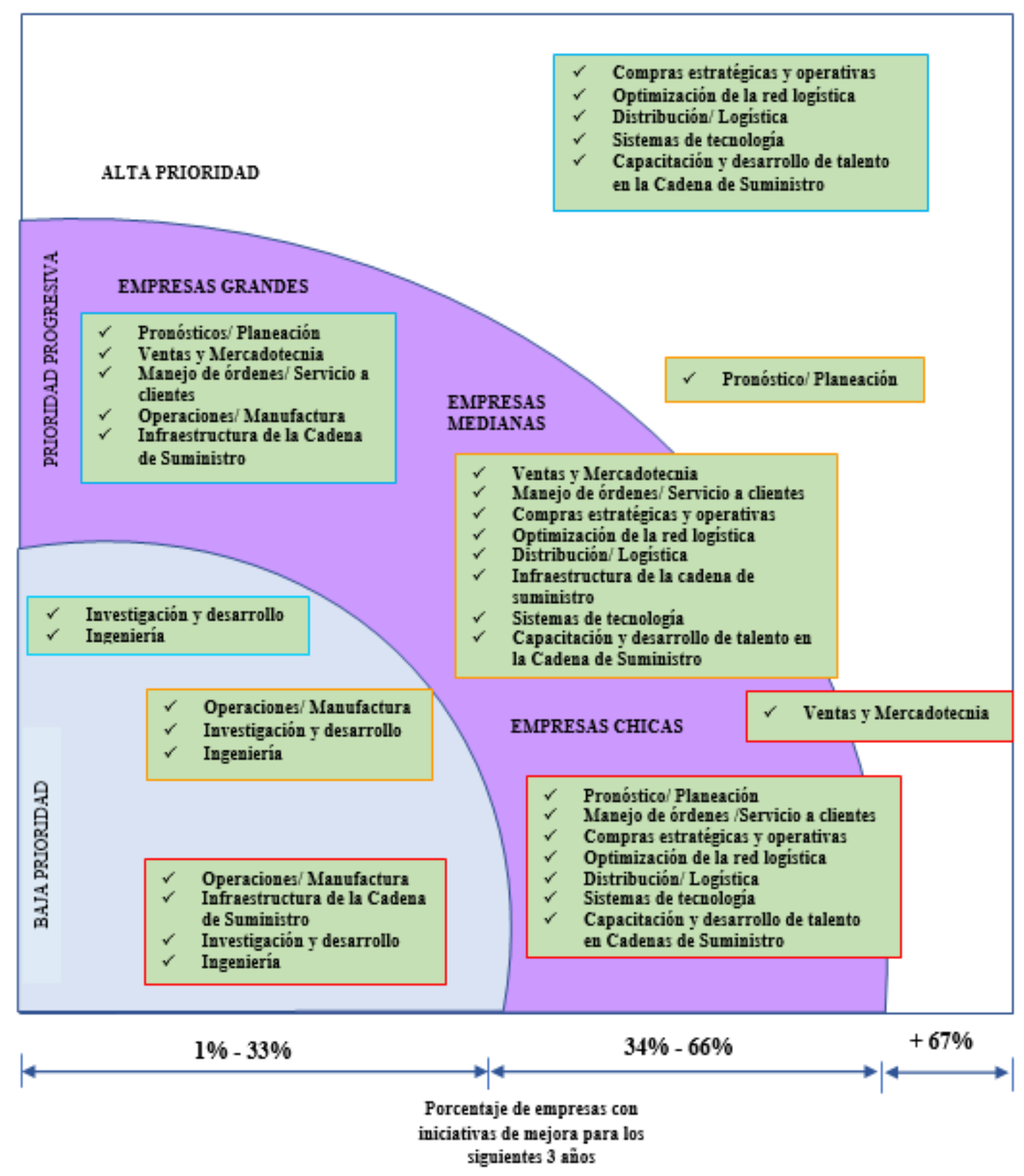

Empresa chica - Menos de \$500 MMXN

Empresa mediana - Entre $\$ 500$ y $\$ 5,000 \mathrm{MMXN}$

Empresa grande- Entre $\$ 5,000$ y $\$ 10,000 \mathrm{MMX}$ 
La figura 1.7 describe las prioridades de las empresas chicas, medianas y grandes, las cuales se diferencian por la logística que emplean. La prioridad de las empresas chicas se basa en una logística de ventas y mercadotecnia, mientras las empresas medianas comprenden una logística de pronóstico/planeación incorporando la infraestructura de cadena de suministro. Por último, las empresas grandes tienen como alta prioridad la ingeniería, la investigación y el desarrollo. En lo general, durante las últimas dos décadas se puede decir que ha habido crecimiento y desarrollo para todas aquellas empresas que han sido capaces de entender la dinámica de los mercados. Y para aquellos proveedores de servicios logísticos que han sido capaces de crecer junto con sus clientes en la región de Norteamérica (TLCAN), contribuyendo al desarrollo de México en este sentido y construyendo en el mercado mexicano relaciones de largo plazo, satisfaciendo las necesidades logísticas de sus clientes y proveyendo valor en diferentes industrias, diferentes geografías dentro de México y en la zona fronteriza con Estados Unidos.

\section{Conclusiones}

A raíz de la apertura comercial de la economía mexicana se ha presentado una demanda creciente del sector exportador e importador del país, así como de otros sectores productivos y de servicios relacionados. Lo que ha implicado establecer políticas de logística eficientes que se encuentran presentes a lo largo de toda la cadena de suministro involucrando tanto al sector público como privado (productores, proveedores y comercializadores), con el propósito de ir transformando la logística nacional en una disciplina más competitiva, productiva, integrada y especializado, en un contexto económico globalizado.

En este sentido, la globalización redefinió las funciones del Estado mexicano como facilitador de factores de infraestructura, educación logística, servicios logísticos, entre otros, para que tanto el sector público como privado se vuelvan internacionalmente competitivos. Sin embargo, existen retos para la economía nacional tales como la mejora continua en las condiciones y características de la infraestructura física (puertos, aeropuertos, carreteras federales, caminos y vialidades locales, entre otros), la adecuación y cumplimiento de las leyes y reglamentos para una logística competitiva, integral y eficiente.

Las políticas de logística y cadena de suministro en México y sus empresas evolucionan en el mundo del comercio exterior pensando holísticamente y actuando de manera local lo que les permite competir y ganar mercados en una economía globalizada, caracterizada por sistemas de producción fragmentados que son manejados a escala mundial por múltiples empresas transnacionales.

\section{Referencias}

Ackoff, R. (2010). El paradigma de Ackoff. Una administración sistémica. México: Limusa

Adriaenséns, Marianela \& Flores, Miguel Ángel. (2004). Introducción a los Negocios en un Mundo Cambiante. Cuarta Edición. México: Mc Graw Hill.

Ballou, Ronald H. (1999). Business Logistics Management. México: Prentice Hall.

Boccara, Nino. (2004). Modeling Complex Systems. New York, USA: Springer-Verlag. Disponible en http://www.fulviofrisone.com/attachments/article/412/modeling\%20complex\%20systems\%20$\% 20$ boccara.pdf

Bohórquez Vásquez, Ella Cecilia \& Puello Fuentes, Roy Alfonso (2013). Diseño de un modelo de gestión logística para mejorar la eficiencia organizacional de la empresa Coralinas \& Pisos S.A. Corpisos S.A. en el Municipio de Turbaco, Bolívar. Colombia: Universidad de Cartagena, Facultad de Ciencias Económicas y Administrativas. Disponible https://www.academia.edu/10602946/DISE\%C3\%91O_DE_UN_MODELO_DE_GESTI\%C3\%93N_L OG\%C3\%8DSTICA_PARA_MEJORAR_LA

Castellanos Ramírez, Andrés (2009). Manual de la gestión logística del transporte y la distribución de mercancías. Barranquilla, Colombia: Ediciones Uninorte. Disponible en https://www.academia.edu/24339874/Manual_de_gestion_logistica_del_transporte_distribucion_de_m ercancias 
Consultoría Deloitte (2017). Eficiencia en la cadena de suministro. Estudio comparativo de prácticas y tendencias en México. México: Socio de Estrategia y Operaciones en Consultoría Deloitte México. Disponible

https://www2.deloitte.com/content/dam/Deloitte/mx/Documents/strategy/SoyLogistico.pdf

Council of Supply Chain Management Professionals (2014). Documento. Disponible en http://cscmp.org/about-us/supply-chain-management-definitions

Lamb, Charles, Hair, Joseph \& McDaniel, Carl. (2002). Marketing. México: International Thomson Editores S.A.

Luttwak, E. A. (1971). Dictionary of Modern War. New York: Harper \& Row,

Franco Vázquez, Pablo César (2008). Aproximación teórica al concepto integral de logística. Revista Región \& Gestión, 6, 65 https://biblioteca.ucp.edu.co/ojs/index.php/gestionyregion/article/viewFile/903/854

Guide, Van Wassenhove (2002). La cadena de suministro. Revista de Negocios Harvard, 80 (2), 25-26.

Jiménez Sánchez, José Elías \& Hernández García, Salvador (2002). Marco conceptual de la cadena de suministro: un nuevo enfoque logístico. Querétaro, México: Secretaría de Comunicaciones y Transportes, Publicación Técnica No. 215. Disponible en https://imt.mx/archivos/Publicaciones/PublicacionTecnica/pt215.pdf

Mora G., Luis Aníbal (2008). Gestión logística Integral. Bogotá: Ecoe Ediciones.

Murguía, Luis (2014). Evolución y crecimiento de la logística en México. Disponible en http://www.logisticamx.enfasis.com/notas/71248-evolucion-y-crecimiento-la-logistica-mexico

Romero Pimentel, Roberto \& Elizondo Cortés, Mayra (2015). Coordinación de la cadena de suministro: de la administración tradicional al enfoque sistémico. En Téllez Vázquez, Sandra, Cedillo Campos, Miguel Gastón \& Jiménez García, José Alfredo. Logística y cadena de suministros: tendencias y desafíos en México. Guanajuato, México: Universidad Politécnica de Guanajuato, 127-144.

Servera-Francés, David (2010). Concepto y evolución de la función logística. Innovar Revista de Ciencias Administrativas y Sociales, 20(38), 271-234. Disponible en https://www.redalyc.org/articulo.oa?id=81819024018

Tzu, Sun (2005). El arte de la guerra. Estado de México: Ediciones Leyenda

USAID/México (2014). Estudio de indicadores del desempeño logístico de las cadenas de suministro en México. México: Secretaria de Economía.

Villafañe Alonso, Pablo (2014). Análisis de la cadena de suministro y su relación con la logística: caso del Centro de Michelin Valladolid. España: Universidad de Valladolid. Disponible en https://uvadoc.uva.es/bitstream/handle/10324/11781/PFM-P-145.pdf?sequence=1

Waters, D. (2007). Global logistics: New Directions in Supply Chain Management. The United Kingdom: Kogan Page Publishers.

Wolfram, Stephen. (2002). A new kind of science. Champaign, IL: Wolfram Media Inc. Disponible en https://www.ams.org/journals/bull/2003-40-01/S0273-0979-02-00970-9/S0273-0979-02-00970-9.pdf

Zuluaga Mazo, Abdul, Gómez Montoya, Rodrigo A. \& Fernández Henao, Sergio A. (2014). Indicadores logísticos en la cadena de suministros como apoyo al modelo scor. Revista Clío América, 8(15), 90-110. Disponible en http://oaji.net/articles/2016/3167-1472232109.pdf 